\begin{tabular}{|c|l|}
\hline Title & Reconstruction of the saframycin core scaffold defines dual Pictet-Spengler mechanisms \\
\hline Author(s) & Koketsu, Kento; Watanabe, Kenji; Suda, Haruna; Oguri, Hiroki; Oikawa, Hideaki \\
\hline Citation & $\begin{array}{l}\text { Nature Chemical Biology, 6(6), 408.410 } \\
\text { https://doi.org/40.1038/hchembio.365 }\end{array}$ \\
\hline Issue Date & $2010-06$ \\
\hline Doc URL & http://hdl.handle.net/2115/44310 \\
\hline Type & article (author version) \\
\hline Additional Information & There are other files related to this item in HUSCAP. Check the above URL. \\
\hline File Information & NCB6-6_408-410.pdf \\
\hline
\end{tabular}

Instructions for use 


\title{
Enzymatic reconstruction of the saframycin core scaffold defines dual Pictet-Spengler mechanisms
}

\author{
Kento Koketsu, ${ }^{1}$ Kenji Watanabe, ${ }^{2}$ Haruna Suda, ${ }^{1}$ Hiroki Oguri ${ }^{1}$ and Hideaki Oikawa ${ }^{1}$ \\ ${ }^{1}$ Division of Chemistry, Graduate School of Science, Hokkaido University, Sapporo \\ 060-0810, Japan \\ ${ }^{2}$ School of Pharmaceutical Sciences, University of Shizuoka, Shizuoka, 422-8526, Japan
}

\begin{abstract}
s (70 words, 3 sentences)
Saframycin A is a potent antitumor antibiotic having unique pentacyclic tetrahydroisoquinoline scaffold. We found that the single nonribosomal peptide synthetase SfmC catalyzes a seven-step transformation from readily synthesized dipeptidyl substrates with long acyl chains into a complex saframysin scaffold. Based on series of enzymatic reactions, we propose a detailed mechanism involving the reduction of various peptidyl thioesters by an identical R-domain followed by C-domain-mediated Pictet-Spengler reactions in an iterative manner.
\end{abstract}


Saframycin A (1) from Streptomyces lavendulae is a representative tetrahydroisoquinoline antibiotic (Fig. 1). ${ }^{1}$ Members of this family have been isolated from various soil bacteria and marine vertebrates, such as sponges and ascidians, ${ }^{2}$ and exhibit potent antitumor activity derived from iminium ions via a carbinolamine moiety and its equivalents. The remarkably potent saframycin analog ecteinascidin 743 (ET-743) has been approved as an anticancer drug for use in patients with soft tissue sarcoma. ${ }^{3}$ Ecteinascidins share the central pentacyclic core with saframycins, except for the oxidation state of their terminal rings and the additional ten-membered macrolactone bridge found in ET-743. Due to the limited supply from natural sources, the production of ET-743 currently relies on a semisynthetic process $^{4}$ requiring more than 25 transformation steps. Biosynthetic engineering ${ }^{5-7}$ may be an ideal alternative strategy for generating this important antibiotic from the viewpoint of efficiency and minimal environmental impact. Therefore, we examined the biosynthetic mechanism by which the pentacyclic tetrahydroisoquinoline scaffold is formed in these antitumor antibiotics.

A series of biosynthetic studies on $\mathbf{1}$ have previously established L-alanine, glycine, and two molecules of the modified amino acid 2, derived from L-tyrosine, as its biosynthetic precursors. ${ }^{8-10}$ To date, three biosynthetic gene clusters of $\mathbf{1}$, safracin $\mathrm{B}$, and saframycin Mx1 have been identified, and analyses using bioinformatics methodologies indicated that the saframycin backbone is constructed by nonribosomal polypeptide synthetases (NRPSs). ${ }^{9,11,12}$ Recent report shows that the saframycin NRPSs (SfmA, SfmB, SfmC) assemble a putative tetrapeptidyl intermediate via an unusual biosynthetic 
mechanism in the SfmC-catalyzed iterative reaction, ${ }^{12}$ and that the subsequent unspecified cyclization reactions with modification enzymes form the pentacyclic skeleton. Although several groups proposed alternative biosynthetic schemes, ${ }^{9,12,13}$ no experimental data have yet been reported regarding biosynthetic intermediates or enzymatic conversions in vitro. Therefore, the detailed biosynthetic mechanism of this intriguing transformation remains unclear.

NRPSs are modular enzymes that generally produce a wide range of small molecule polypeptides with established reaction sequence. ${ }^{14}$ However, the structure of $\mathbf{1}$ lacks several carbonyl groups on the peptide backbone. Therefore, textbook knowledge cannot be applied to the saframycin NRPSs, and a novel mechanism must be involved in construction of the unique cyclic system. Here, we report the remarkable reaction mechanism of SfmC, which catalyzes seven transformation steps with sophisticated communication between the individual domains.

Many lipopeptides, such as mycosubtilin, daptomycin, and friulimicin, possess a long fatty acyl chain at their $N$-termini. ${ }^{15,16}$ The characteristics of the NRPSs responsible for producing these lipopeptides include a fatty acid-loading system, such as acyl-CoA ligase (AL), an acyl carrier protein (ACP), and the distinctive $N$-terminal condensation (C) domain of the first module of NRPS.${ }^{15}$ Homologous AL-ACP didomains are present in the gene clusters of 1 (S. lavendulae) and saframycin Mx1 (Myxococcus xanthus), although their structures lack such fatty acyl chains. Although these domains have previously been overlooked as participating in the reaction pathway, we speculated that this cryptic long acyl chain ${ }^{17-19}$ may play a crucial role in saframycin biosynthesis. Based on the organization 
of the second and third modules, which include the adenylation (A) domains specific for L-alanine and glycine, respectively, the NRPS-catalyzed reactions of the first three modules should yield an $N$-acyldipeptidyl intermediate 3 (Fig. 1). This leaves the last module, corresponding to $\mathrm{SfmC}$, to catalyze the remaining considerable transformation from this intermediate to the pentacyclic skeleton.

To verify this hypothesis, we cloned and overexpressed $s f m C$ encoding the last module consisting of the tetradomain C-A-peptidyl carrier protein (PCP)-reduction $(\mathrm{R})$, in Escherichia coli as $\mathrm{N}$ - and $\mathrm{C}$-terminal $\mathrm{His}_{6}$-fusions (Supplementary Fig. 1a). Treatment of the purified apo-SfmC with recombinant Bacillus subtilis 4'-phosphopantetheinyltransferase (PPase) Sfp and coenzyme A provided the holo-SfmC. (Supplementary Fig. 2). Blue native PAGE analysis indicated that this enzyme existed as a homodimer (Supplementary Fig. 1b - 1d). The synthetic putative intermediate analog $N$-myristoyldipeptidyl-S-CoA 4b (Supplementary Fig. 3) was subjected to recombinant SfmC-catalyzed conversion in the presence of $\mathbf{2}, \mathrm{ATP}, \mathrm{Mg}^{2+}$, and NADPH (Fig. 2). In the reaction with $\mathbf{4 b}$, LC-MS analysis of the reaction products indicated that four new products were generated, including the pentacyclic saframycin-type product $\mathbf{5 b}$, as well as three incomplete products 7b, 8b, and 9b (Fig. 2 and Supplementary Fig. 4). The structures of $\mathbf{8 b}$ and $\mathbf{9 b}$ were identical to those of the synthetic bicyclic tetrahydroisoquinoline product and the dipeptidylaldehyde (Supplementary Fig. 4), whereas $\mathbf{7 b}$ was readily converted into $\mathbf{8 b}$ by treatment with $\mathrm{NaBH}_{4}$. As $\mathbf{8 b}$ was presumably derived from the corresponding $\mathbf{7 b}$ by enzymatic overreduction, $\mathbf{7 b}$ and $\mathbf{9 b}$ were regarded as biosynthetic intermediates. To unambiguously elucidate the structure of $\mathbf{5 b}$, we performed an enzymatic reaction with the 
proposed intermediate $\mathbf{9 b}$ in place of $\mathbf{4 b}$. The SfmC-catalyzed conversion with $\mathbf{9 b}$ proceeded efficiently, leading to $\mathbf{5 b}$, and resulted in a marked acceleration of more than tenfold in comparison with the reaction employing 4b (Supplementary Fig. 6). We additionally characterized the cyanoadduct $\mathbf{6}$ - created in a large-scale incubation followed by treatment with $\mathrm{KCN}$ - by various spectroscopic methods, including 2D-NMR analysis (Supplementary Fig. 7, Table 2). The relative stereochemistry of $\mathbf{6}$, as a proxy for $\mathbf{5 b}$, which we named $N$-myristoylpresaframycin, was consistent with that of natural 1 (Fig. 1). It should also be noted that use of the coenzyme NADH significantly improved the production of $\mathbf{5 b}$ up to a $23 \%$ overall yield based on $\mathbf{9 b}$, while the reaction utilizing NADPH resulted in the predominant formation of $\mathbf{8 b}$ over $\mathbf{5 b} \quad(\mathbf{5 b}: \mathbf{8 b}=1: 2)$ (Supplementary Fig. 6).

Based on the results of the SfmC-catalyzed reaction, we proposed a plausible mechanism for assembly of the pentacyclic tetrahydroisoquinoline skeleton from $\mathbf{4 b}$ (Fig. 1). The detection of $\mathbf{7 b}$ and $\mathbf{9 b}$ indicated that the pentacyclic skeleton is assembled in a stepwise manner. The growing polypeptide chains appended to PCP3 on SfmB and to PCP4 on SfmC could interact with the R-domain, which reductively releases $\mathbf{5 b}, \mathbf{7 b}, \mathbf{9 b}$ and $\mathbf{1 0}$ as in the case of pikromycin thioesterase (Pik-TE) domain. ${ }^{20}$ This mechanism explains not only the unusual reduction of the $\mathrm{N}$-acyldipeptidyl intermediate but also the iterative use of the last module. Interestingly, this biosynthetic mechanism is closely related to that in the total synthesis reported by Myers and Kung. ${ }^{21}$

We further examined the role of the acyl chain with a series of synthetic precursors $4 \mathbf{a}-\mathbf{e}$ and $9 \mathbf{a}-\mathbf{c}$. In contrast to the result with $\mathbf{4 b}$, no reduction or cyclization 
products were observed in the case of the $N$-acetyl and the non-acylated CoAs $\mathbf{4 d}, \mathbf{4 e}$. In the experiments with CoA-esters and NADPH (3 mM), 4a and $\mathbf{4 b}$ with longer acyl chains were efficiently converted into $\mathbf{5 a}$ and $\mathbf{5 b}$ but the product ratios - and especially the amounts of overreduction products - were changed by the use of different substrates (Supplementary Figs. 4, 8 and 9). These products were not observed in the experiments of 4a - 4c with NADH. Similar results were obtained in the reactions with dipeptidylaldehydes (Supplementary Fig. 10). Interestingly, the reaction with $N$-lauroylpeptidylaldehyde 9c gave products $5 \mathbf{c}, 7 \mathbf{c}$ and $8 \mathbf{c}$ although the corresponding CoA-ester $\mathbf{4 c}$ did not, indicating that the acyl chain is important for the substrate recognition of the first reduction (Supplementary Figs. 9 and 10).

To probe the stereospecificity of the R-domain reaction, the $\mathrm{SfmC}$-catalyzed reactions in the presence of $(S)$ - and $(R)-\left[4-^{2} \mathrm{H}\right] \mathrm{NADPH}$ were performed and analyzed by LC-MS. Only the experiment with $(S)-\left[4{ }^{2} \mathrm{H}\right] \mathrm{NADPH}$ showed the 3 Da-shifted fragment ion of $\mathbf{5 b}$ thus obtained, as compared with the corresponding ion from the non-labeled $\mathbf{5} \mathbf{b}$ (Supplementary Fig. 11), indicating that the deuterons on the three $\mathrm{sp}^{3}$ carbon centers of 5b are introduced (Fig. 1). The combined experimental evidence thus shows that the SfmC R-domain has unusually broad substrate specificity and mediates the reduction of three distinct thioesters bound to PCPs into the corresponding aldehydes, yet maintains enough specificity to discriminate between the long acyl chains of $\mathbf{4 a}$ and $\mathbf{4 b}$ as compared to $\mathbf{4 d}$ or 4e. The full description of how this cryptic longer acyl chain influences substrate recognition remains to be seen.

To better understand the cofactor preferences of the reaction, we performed the 
$\mathrm{SfmC}$ reaction in several time course experiments using either $\mathrm{NADH}$ or NADPH at different concentrations. A recent report on absolute metabolite concentrations in Escherichia coli showed that intracellular concentrations of NADPH and NADH are 120 and $86 \mu \mathrm{M}$, respectively. ${ }^{22}$ At the plausible physiological NADPH concentration $(200 \mu \mathrm{M})$, the reaction with afforded $\mathbf{5 b}$ as a predominant product while $\mathbf{5 b}$ production was suppressed and accompanied with aberrant by-product formation at $2 \mathrm{mM}$ NADPH (Supplementary Fig. 12). In the experiment with NADH $(200 \mu \mathrm{M}), \mathbf{5 b}$ formation was below $1 \mu \mathrm{M}$ while at the high concentration $(2 \mathrm{mM})$ formation of $\mathbf{5 b}$ was increased to 18 $\mu \mathrm{M}$ (Supplementary Fig. 12). In addition, LC-MS data of the products in the competition experiments with $S-\left[4-^{2} \mathrm{H}\right] \mathrm{NADPH}$ and NADH $(100 \mu \mathrm{M}$ each) showed shifted molecular ion peaks at $\mathrm{m} / \mathrm{z} 551.5$ for $\mathbf{7 b}, 707.6$ for $\mathbf{5 b}$ and 536.5 for $\mathbf{8 b}$ while the products in the experiments with NADPH and $S-\left[4-{ }^{2} \mathrm{H}\right] \mathrm{NADH}(100 \mu \mathrm{M}$ each) gave no shifted peak, showing that SfmC uses NADPH preferentially (Supplementary Fig. 13). However, at high concentration (1 $\mathrm{mM}$ each) both labeled cofactors gave shifted peaks of all products, indicating that both cofactors were used with nearly equal efficiency. This suggests that efficient production is artificial event.

Finally, to determine the domain responsible for mediating the Pictet-Spengler reaction, we prepared two mutants (C-domain deletion: A-PCP-R, R-domain deletion: C-A-PCP) of SfmC (Supplementary Fig. 1). The enzymatic reaction of $N$-myristoyldipeptidyl-CoA $\mathbf{4 b}$ with the C-domain deletion mutant simply yielded $\mathbf{9 b}$, but no cyclization product (Supplementary Fig. 14). Moreover, the reaction of 9b with the R-domain deletion mutant, followed by reduction with $\mathrm{NaBH}_{4}$, gave $\mathbf{8 b}$ (Supplementary 
Fig. 15), whereas none of this product was obtained in the reaction of $9 \mathbf{b}$ with the C-domain deletion mutant. These observations indicated that this single-turnover reaction with the R-domain deletion mutant generates the Pictet-Spengler product bound to the PCP4 domain, which is reductively released with $\mathrm{NaBH}_{4}$. Therefore, we conclude that the intriguing Pictet-Spengler reaction is catalyzed by the C-domain, designated here as the "PS-domain." Additional function of C-domain is known in a Cy-domain which catalyzes similar multistep transformation including peptide bond formation and heterocycle formation between amide carbonyl and adjacent $\mathrm{OH}$ or $\mathrm{SH}$ group. ${ }^{23}$ Sequence alignment and phylogenetic analyses of PS-domains showed modest identity (13\% - 25\%) to other Cand Cy-domains (Supplementary Figs. 16 and 17). Among the core consensus sequences of the C-domain, the highly conserved motif HHxxxDG in core 3 which plays important role for catalysis of condensation, ${ }^{23}$ is replaced with the motif $(\operatorname{HxxxxD})$ in the PS-domains, suggesting that this difference may be significant for the Pictet-Spengler reaction.

In conclusion, we have elucidated the detailed biosynthetic mechanism involved in the construction of saframycin pentacyclic tetrahydroisoquinoline scaffold, which involve sophisticated communication of the $\mathrm{SfmC}$ domains as well as the cryptic roles of the fatty acyl chain. The successful production of $\mathbf{5 b}$ with SfmC from simple starting materials would set the stage for chemoenzymatic synthesis generating natural product analogs, including advanced intermediates in the semisynthesis of ecteinascidin $743 .{ }^{4}$ 


\section{References}

1. Arai, T., Takahashi, K. \& Kubo, A. New antibiotics saframycins A, B, C, D and E. J. Antibiot. 30, 1015-1018 (1977).

2. Scott, J. D. \& Williams, R. M. Chemistry and biology of the tetrahydroisoquinoline antitumor antibiotics. Chem. Rev. 102, 1669-1730 (2002).

3. Cuevas, C. \& Francesch, A. Development of Yondelis (trabectedin, ET-743). A semisynthetic process solves the supply problem. Nat. Prod. Rep. 26, 322-337 (2009).

4. Cuevas, C. et al. Synthesis of ecteinascidin ET-743 and phthalascidin Pt-650 from cyanosafracin B. Org. Lett. 2, 2545-2548 (2000).

5. Watanabe, K. et al. Total biosynthesis of antitumor nonribosomal peptides in Escherichia coli. Nat. Chem. Biol. 2, 423-428 (2006).

6. Watanabe, K. et al. Escherichia coli allows efficient modular incorporation of newly isolated quinomycin biosynthetic enzyme into echinomycin biosynthetic pathway for rational design and synthesis of potent antibiotic unnatural natural product. J. Am. Chem. Soc. 131, 9347-9353 (2009).

7. Watanabe, K. et al. Rationally engineered total biosynthesis of a synthetic analogue of a natural quinomycin depsipeptide in Escherichia coli. ChemBioChem 10, 1965-1968 (2009).

8. Mikami, Y. et al. Biosynthetic studies on saframycin A, a quinone antitumor antibiotic produced by Streptomyces lavendulae. J. Biol. Chem. 260, 344-348 (1985). 
9. Velasco, A. et al. Molecular characterization of the safracin biosynthetic pathway from Pseudomonas fluorescens A2-2: designing new cytotoxic compounds. Mol. Microbiol. 56, 144-154 (2005).

10. Fu, C. Y. et al. Biosynthesis of 3-hydroxy-5-methyl-O-methyltyrosine in the saframycin/ safracin biosynthetic pathway. J. Microbiol. Biotechnol. 19, 439-446 (2009).

11. Pospiech, A., Bietenhader, J. \& Schupp, T. Two multifunctional peptide synthetases and an $O$-methyltransferase are involved in the biosynthesis of the DNA-binding antibiotic and antitumour agent saframycin Mx1 from Myxococcus xanthus. Microbiology 142, 741-746 (1996).

12. Li, L. et al. Characterization of the saframycin A gene cluster from Streptomyces lavendulae NRRL 11002 revealing a nonribosomal peptide synthetase system for assembling the unusual tetrapeptidyl skeleton in an iterative manner. J. Bacteriol. 190, $251-263$ (2008).

13. Schmidt, E. W., Nelsona, J. T. \& Fillmorea, J. P. Synthesis of tyrosine derivatives for saframycin MX1 biosynthetic studies. Tetrahedron Lett. 45, 3921-3924 (2004).

14. Fischbach, M. A. \& Walsh, C. T. Assembly-line enzymology for polyketide and nonribosomal Peptide antibiotics: logic, machinery, and mechanisms. Chem. Rev. 106, 3468-3496 (2006).

15. Baltz, R. H., Miao, V.\& Wrigley, S. K. Natural products to drugs: daptomycin and related lipopeptide antibiotics. Nat. Prod. Rep. 22, 717-741 (2005). 
16. Hansen, D. B., Bumpus, S. B., Aron, Z. D., Kelleher, N. L.\& Walsh, C. T. The loading module of mycosubtilin: an adenylation domain with fatty acid selectivity. $J$. Am. Chem. Soc. 129, 6366-6367 (2007).

17. Du, L., Sanchez, C., Chen, M., Edwards, D. J. \& Shen, B. The biosynthetic gene cluster for the antitumor drug bleomycin from Streptomyces verticillus ATCC15003 supporting functional interactions between nonribosomal peptide synthetases and a polyketide synthase. Chem. Biol. 7, 623-642 (2000).

18. Mossialos, D. et al. Identification of new, conserved, non-ribosomal peptide synthetases from fluorescent pseudomonads involved in the biosynthesis of the siderophore pyoverdine. Mol. Microbiol. 45, 1673-1685 (2002).

19. Visca, P., Imperi, F. \& Lamont, I. L. Pyoverdine siderophores: from biogenesis to biosignificance. Trends Microbiol. 15, 22-30 (2007).

20. Xue, Y.\& Sherman, D. H. Alternative modular polyketide synthase expression controls macrolactone structure. Nature 403, 571-575 (2000).

21. Myers, A. G. \& Kung, D. W. A concise, stereocontrolled synthesis of (-)-saframycin A by the directed condensation of $\alpha$-amino aldehyde precursors. J. Am. Chem. Soc. 121, 10828-10829 (1999).

22. Bennett, B. D. et al. Absolute metabolite concentrations and implied enzyme active site occupancy in Escherichia coli. Nat. Chem. Biol. 5, 593-599 (2009).

23. Duerfahrt, T., Eppelmann, K., Muller, R. \& Marahiel, M. A. Rational design of a bimodular model system for the investigation of heterocyclization in nonribosomal peptide biosynthesis. Chem. Biol. 11, 261-271 (2004). 


\section{AUTHOR CONTRIBUTIONS}

H. Oikawa and K.W. designed the research, and H. Oikawa wrote the manuscript. K.K. and

H.S. synthesized the substrates for the enzymatic reactions. K.W. cloned the $s f m C$ gene and optimized the expression conditions. K.K. prepared the deletion enzymes and performed all enzymatic reactions. H. Oguri advised on the research and made a significant contribution to a proposal of the reaction mechanism.

Competing interest statement. The authors declare no competing financial interests.

\section{Figure legends}

Figure 1. Proposed overall scheme for the pentacyclic core skeleton assembly. The reactions proceed in the following sequence: (1) The 1st reduction of the thioester $\mathbf{4 b}$. (2)

The 1st loading of 2. (3) The 1st Pictet-Spengler reaction. (4) The 2nd reduction of the thioester intermediate. (5) The 2nd loading of 2. (6) The 2nd Pictet-Spengler reaction. (7) The 3rd reduction of the thioester intermediate. The asterisks $(*)$ in $\mathbf{5 b}$ indicate the 
deuterated positions in the experiment with $(S)-\left[4-{ }^{2} \mathrm{H}\right] \mathrm{NADPH}$.

Figure 2. (125 words) SfmC-catalyzed reaction with the various dipeptidyl-CoA substrates 4a - 4e. (a) The scheme depicts the SfmC-catalyzed conversion of $\mathbf{4 b}$ and $\mathbf{2}$ to the pentacyclic skeleton $\mathbf{5 b}$, the bicyclic aldehyde $\mathbf{7 b}$, the bicyclic alcohol $\mathbf{8 b}$ and the $N$-myristoyldipeptidylaldehyde $\mathbf{9 b}$. (b) ESI-MS (inset) and ESI-MS/MS spectra of $\mathbf{5 b}$. (c) ESI-MS (inset) and ESI-MS/MS spectra of 7b. (d) ESI-MS (inset) and ESI-MS/MS spectra of $8 \mathbf{b}$. 


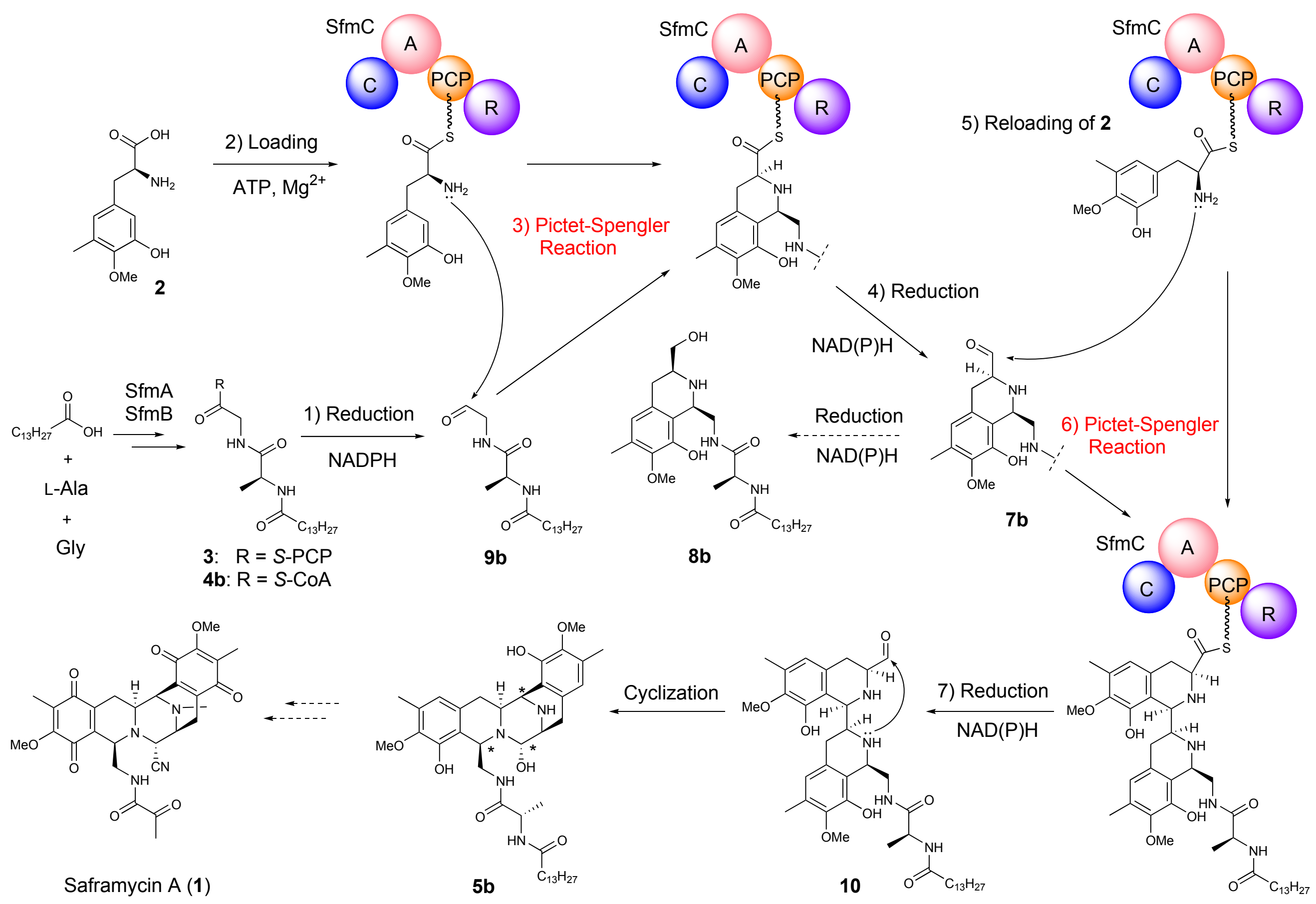


